\title{
Case of Pyoderma Gangrenosum Associated with Ulcerative Colitis Successfully Treated with Infliximab
}

\author{
*Tomotaka Mabuchi, Hanako Yamaoka \\ Department of Dermatology, Tokai University School of Medicine, 143 Shimokasuya, \\ Isehara, Kanagawa, Japan \\ mabuchi@is.icc.u-tokai.ac.jp
}

\begin{abstract}
Pyoderma gangrenosum $(P G)$ is a rare, ulcerating inflammatory skin disease. $P G$ is often associated with systemic diseases, including inflammatory bowel disease (IBD). Herein, we report a case of $P G$ associated with ulcerative colitis successfully treated with infliximab. Our case report supports the efficacy of infliximab for refractory case of PG with IBD.
\end{abstract}

Keywords: pyoderma gangrenosum, inflammatory bowel disease, infliximab

\section{INTRODUCTION}

The pathogenesis of pyoderma gangrenosum (PG) is still poorly understood, but, is thought to be a multifactorial combination of inflammatory mediators, neutrophilic dysfunction, and genetic predisposition [1]. PG is often associated with systemic diseases, including inflammatory bowel disease (IBD). So far, systemic corticosteroids are regarded as first-line therapy. Besides this, tumor necrosis factor (TNF) $\alpha$ inhibitors are proposed as effective therapies [1].

\section{CASE REPORT}

A 61-year-old Japanese male was diagnosed with PG associated ulcerative colitis (UC) with vesicointestinal fistula three months before visiting our outpatient clinic. Neither of the symptoms showed any response to the following treatments: $4,000 \mathrm{mg} / \mathrm{day}$ of oral and $1 \mathrm{~g} /$ day of suppository of mesalazine, five times leukocytapheresis (LCAP) but not granulocyte and monocyte adsorption apheresis (GMA), and intravenous administration of $40 \mathrm{mg}$ of prednisolone. Thus, he was moved to gastroenterology department of Tokai University Hospital to treat with other options. We noticed multiple, large, progressive, and painful skin ulcers with yellowish necrosis on his legs (Figure 1). Laboratory test findings were as follows: white blood cell count, $6,000 / \mu \mathrm{L}$; C-reactive protein, $4.44 \mathrm{mg} / \mathrm{dL}$; albumin, $1.9 \mathrm{~g} / \mathrm{dL}$; creatinine, $1.01 \mathrm{mg} / \mathrm{dL}$; blood urea nitrogen, $24 \mathrm{mg} / \mathrm{dL}$; aspartate aminotransferase, $79 \mathrm{U} / \mathrm{L}$; alanine aminotransferase, 473 $\mathrm{U} / \mathrm{L} ; \quad \gamma$-glutamyl transpeptidase, $262 \mathrm{U} / \mathrm{L} ;$ alkaline phosphatase, $1272 \mathrm{U} / \mathrm{L}$; erythrocyturia, albuminuria, and pneumaturia. Histopathological examination showed spongiosis and abscess of the epidermis, marked lymphocytic and neutrophilic infiltration with focal abscess formation, and perivascular fibrin deposition of the dermis (Figure 2). These findings were consistent with PG. After intravenous administrations of $5 \mathrm{mg}$ of infliximab at week 0 and 2, skin ulcers as well as digestive symptoms rapidly responded. The skin symptoms healed with scar formation at week 3 (Figure 3). As a result, systemic prednisolone was gradually reduced. Infliximab infusions are continued as maintenance therapy.

\section{Discussion}

In addition to a number of case reports of $P G$ responding to infliximab [1,2], a rapid efficacy of $5 \mathrm{mg} / \mathrm{kg}$ of infliximab for PG with or without IBD was supported by a randomized, double-blind, placebo-control study in 2006 [3]. 30 patients were enrolled in this study. 6 of $13(46 \%)$ and 1 of $17(6 \%)$ patients received infliximab and placebo had improved at week 2 , respectively. At week 2, the 23 non-responders were offered open labelled infliximab. Namely, 29 patients received infliximab. As a result, 20 of 29 patients (69\%), including 3 of 7 patients who received two doses of infliximab at week 0 and 2, had improved by week 4 . At week 6,6 patients (21\%) were in complete 
remission, but, 9 patients (31\%) were non-responders. Another small clinical trial of infliximab for PG with IBD was completed in 2010 in USA, but the results are not yet published. Abundant expression of TNF $\alpha$ in stromal neutrophils and macrophages, and migrating keratinocytes in PG skin was reported [4], but, exact role of TNF $\alpha$ in the pathogenesis of $P G$ is still unclear. It is likely to play as one of the key pro-inflammatory cytokines. IL-8, whose secretion is also induced by TNF $\alpha$, represents a potent chemoattractant for neutrophils.

In conclusion, if a patient with PG has current IBD, infliximab should be considered as one of the effective therapeutic options.

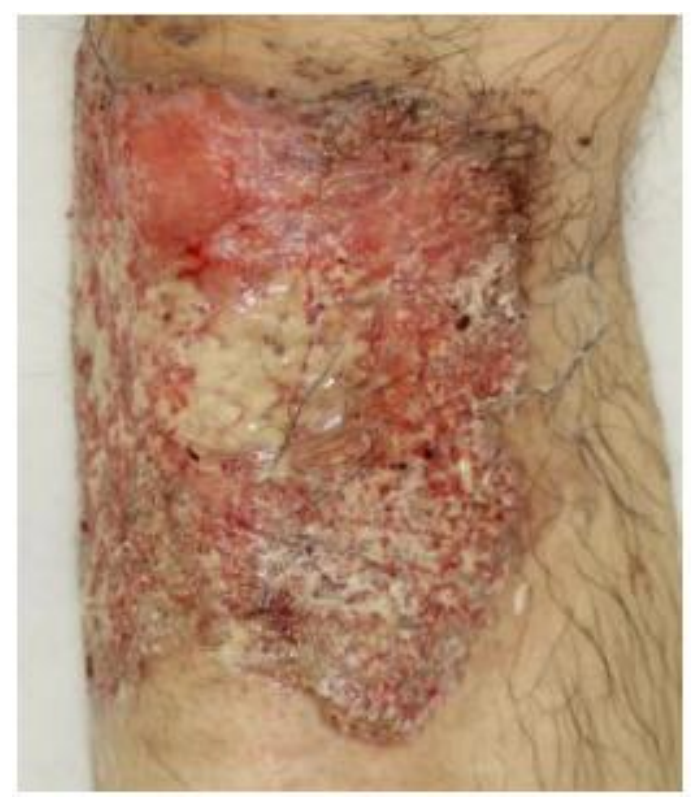

Figure1. Multiple, large, progressive, and painful skin ulcers with yellowish necrosis on his legs were noticed.

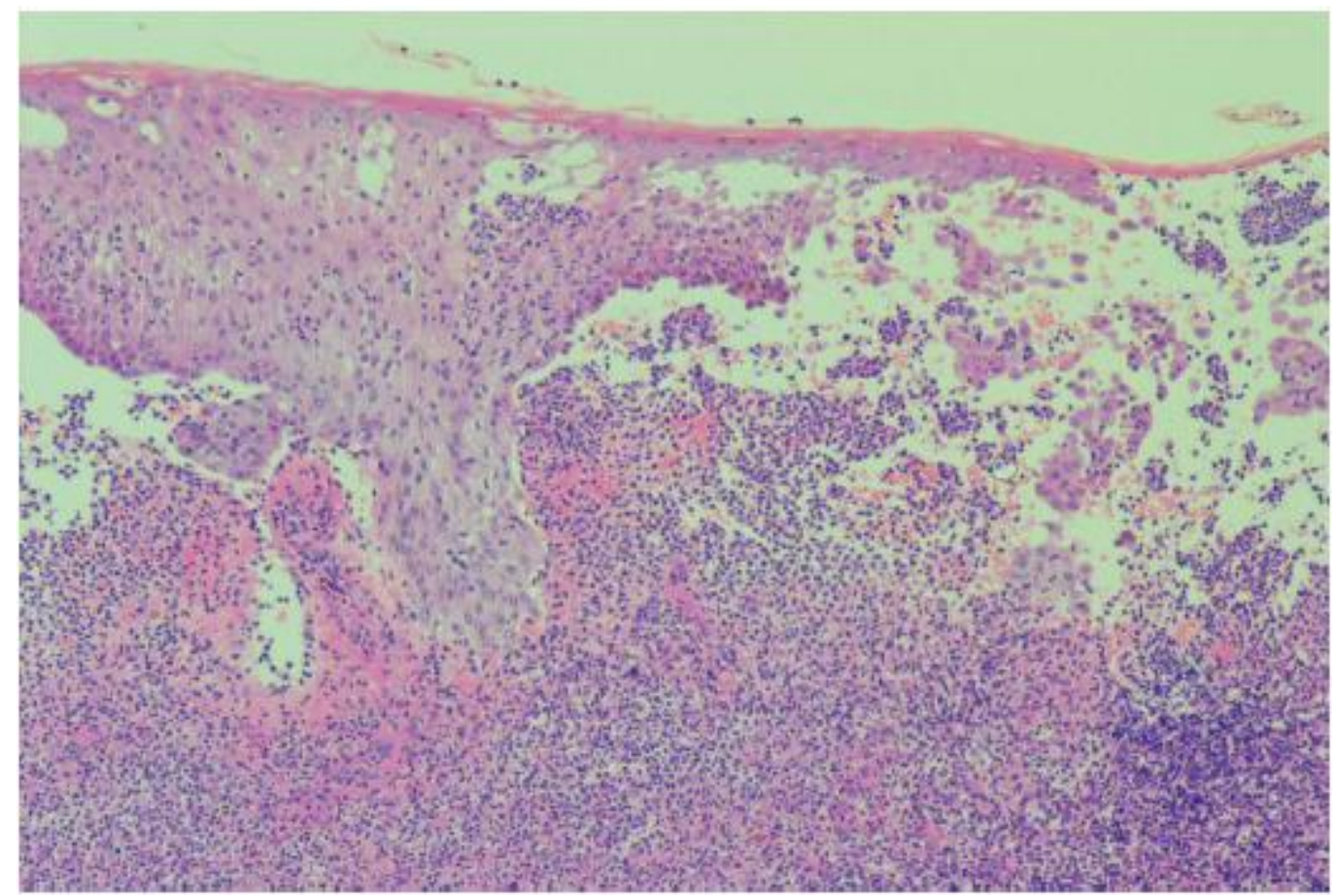

Figure2. Histopathological examination showed spongiosis, marked lymphocytic and neutrophilic infiltration with focal abscess formation, and perivascular fibrin deposition (HE stain, original magnification $\times 100$ ). 


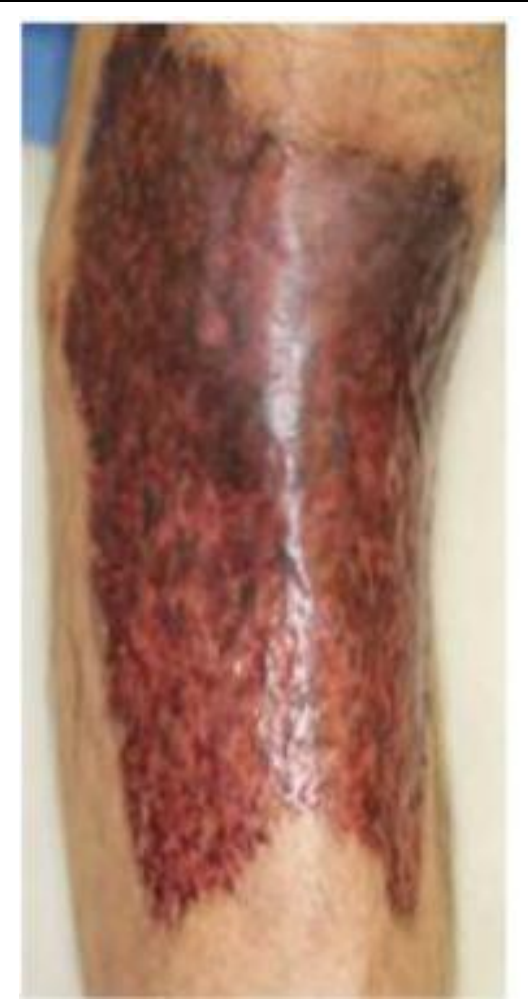

Figure3. The skin symptoms rapidly responded to infliximab infusions.

\section{REFERENCES}

[1] Braswell SF, Kostopoulos TC, Ortega-Loayza AG. Pathophysiology of pyoderma gangrenosum (PG): an updated review. J Am Acad Dermatol. 2015; 73:691-698.

[2] Patel F, Fitzmaurice S, Duong C, He Y, Fergus J, Raychaudhuri SP, et al. Effective strategies for the management of pyoderma gangrenosum: a comprehensive review. Acta Derm Venereol. 2015; 95:525-531.

[3] Brooklyn TN, Dunnill MG, Shetty A, Bowden JJ, Williams JD, Griffiths CE, et al. Infliximab for the treatment of pyoderma gangrenosum: a randomised, double blind, placebo controlled trial. Gut. 2006; 55:505-509.

[4] Bister V, Makitalo L, Jeskanen L, Saarialho-Kere U. Expression of MMP-9, MMP-10 and TNF-alpha and lack of epithelial MMP-1 and MMP-26 characterize pyoderma gangrenosum. J Cutan Pathol. 2007; 34:889-898. 